\title{
Some Asymptotics for Extremal Polynomials
}

\author{
Gökalp Alpan, Alexander Goncharov, and Burak Hatinoğlu
}

\begin{abstract}
We review some asymptotics for Chebyshev polynomials and orthogonal polynomials. Our main interest is in the behaviour of Widom factors for the Chebyshev and the Hilbert norms on small sets such as generalized Julia sets.
\end{abstract}

\subsection{Introduction}

Let $K \subset \mathbb{C}$ be a compact set containing an infinite number of points and $\operatorname{Cap}(K)$ stand for the logarithmic capacity of $K$. Given $n \in \mathbb{N}$, by $\mathscr{M}_{n}$ we denote the set of all monic polynomials of degree at most $n$.

Given probability measure $\mu$ with $\operatorname{supp}(\mu)=K$ and $1 \leq p \leq \infty$, we define the $n$th Widom factor associated with $\mu$ as $W_{n}^{p}(\mu)=\frac{\inf _{Q \in \mathscr{M} n}\|\bar{Q}\|_{p}}{(\operatorname{Cap}(K))^{n}}$ where $\|\cdot\|_{p}$ is taken in the space $L^{p}(\mu)$. If $K$ is polar, then let $W_{n}^{p}(\mu):=\infty$. Clearly, $W_{n}^{p}(\mu) \leq W_{n}^{r}(\mu)$ for $1 \leq p \leq r \leq \infty ; W_{n}^{p}$ is invariant under dilation and translation of $\mu$.

We omit the upper index for the case $p=\infty$. Here the values $W_{n}(K)=\frac{\left\|T_{n, K}\right\|_{\infty}}{(\operatorname{Cap}(K))^{n}}$ provide us with information about behaviour of the Chebyshev polynomials $T_{n, K}$ on $K$. In Sect. 7.2 we review some results in this direction.

Another important case is $p=2$, where $\inf _{\mathscr{M}_{n}}\|Q\|_{2}$ is realized on the monic orthogonal polynomial with respect to $\mu$. The sequence $\left(W_{n}^{2}(\mu)\right)_{n=1}^{\infty}$ is rather convenient to describe measures that are regular in the Stahl-Totik sense and the Szegó class that provides the strong asymptotics of general orthogonal polynomials. In Sect. 7.3 we recall basic concepts of the theory, in Sect. 7.4 model examples of $W_{n}^{2}(\mu)$ are considered. The next sections are related to the results of the first two authors about orthogonal polynomials with respect to equilibrium measures on generalized Julia sets. All results of the authors mentioned in this review were recently published or submitted except Theorem 7.1, which is new.

G. Alpan • A. Goncharov ( ()

Department of Mathematics, Bilkent University, Ankara, Turkey

e-mail: gokalp@fen.bilkent.edu.tr; goncha@fen.bilkent.edu.tr

B. Hatinoğlu

Texas A\&M University, College Station, TX, USA

e-mail: burakhatinoglu@mail.math.tamu.edu 
We suggest the name Widom factor for $W_{n}^{p}(\mu)$ because of the fundamental paper [42], where Widom systematically considered the corresponding ratios for finite unions of smooth Jordan curves and arcs.

For basic notions of logarithmic potential theory we refer the reader to [30], log denotes the natural logarithm, $\mu_{K}$ is the equilibrium measure of $K$. Introduction to the theory of general orthogonal polynomials can be found in [33, 34, 37, 40], see [27] for basic concepts of complex dynamics and [13] for a generalization of Julia sets. The symbol $\sim$ denotes the strong equivalence: $a_{n} \sim b_{n}$ means that $a_{n}=$ $b_{n}(1+o(1))$ for $n \rightarrow \infty$.

\subsection{Widom Factors for the Sup-Norm}

Given $K$ as above, by $T_{n, K}$ we denote the $n$th Chebyshev polynomial and by $t_{n}(K)$ the corresponding Chebyshev number $t_{n}(K):=\left\|T_{n, K}\right\|_{\infty}$. By M. Fekete and G. Szegő we have $t_{n}(K)^{\frac{1}{n}} \rightarrow \operatorname{Cap}(K)$ as $n \rightarrow \infty$. Bernstein-Walsh inequality (see, e.g., Theorem 5.5.7 in [30]) implies that $t_{n}(K) \geq(\operatorname{Cap}(K))^{n}$ for all $n$. Thus, $W_{n}(K) \geq 1$ and $\left(W_{n}(K)\right)_{n=1}^{\infty}$ have subexponential growth (that is, $\log W_{n} / n \rightarrow 0$ ). We mention two important cases: $W_{n}(\partial \mathbb{D})=1$ and $W_{n}([-1,1])=2$ for all $n \in \mathbb{N}$.

If $K$ is a subarc of the unit circle with angle $2 \alpha$, then $W_{n}(K) \sim 2 \cos ^{2}(\alpha / 4)$ (see, e.g., p. 779 in [36]). The circle and the interval can be considered now as limit cases with $\alpha \rightarrow \pi$ and $\alpha \rightarrow 0$.

By Schiefermayr [31], $W_{n}(K) \geq 2$ if $K$ lies on the real line.

The behaviour of $\left(W_{n}(K)\right)_{n=1}^{\infty}$ may be rather irregular, even for simple compact sets. Achieser considered in $[1,2]$ the set $K=[a, b] \cup[c, d]$ and showed that $\left(W_{n}(K)\right)_{n=1}^{\infty}$ has a finite number of accumulation points from which the smallest is 2 provided $K$ is a polynomial preimage of an interval. Otherwise, the accumulation points of $\left(W_{n}(K)\right)_{n=1}^{\infty}$ fill out an entire interval of which the left endpoint is 2 .

In the generalization of this result the concept of Parreau-Widom sets is important. Let $K \subset \mathbb{R}$ be regular with respect to the Dirichlet problem. Then the Green function $g_{\mathbb{C} \backslash K}$ of $\mathbb{C} \backslash K$ with pole at infinity is continuous throughout $\mathbb{C}$. By $\mathscr{C}$ we denote the set of critical points of $g_{\mathbb{C} \backslash K}$, where its derivative vanishes. Clearly, $\mathscr{C}$ is at most countable. Then $K$ is called a Parreau-Widom set if

$$
\mathrm{PW}(K):=\sum_{z \in \mathscr{C}} g_{\mathbb{C} \backslash K}(z)<\infty
$$

It was shown recently in [18] that $W_{n}(K) \leq 2 \exp (\mathrm{PW}(K))$ for a Parreau-Widom set $K$.

In extension of Widom's theory, Totik and Yuditskii considered in [39] the case when $K=\cup_{j=1}^{p} K_{j}$ is a union of $p$ disjoint $C^{2+}$ Jordan curves which are symmetric with respect to the real line. They showed that the accumulation points of $\left(W_{n}(K)\right)_{n=1}^{\infty}$ lie in $[1, \exp (\mathrm{PW}(K))]$. Moreover, if the values $\left(\mu_{K}\left(K_{j}\right)\right)_{j=1}^{p}$ are 
rationally independent, then the limit points of $W_{n}(K)$ fill out the whole interval above. We recall that $\left(x_{j}\right)_{j=1}^{n} \subset \mathbb{R}$ are rationally independent if $\sum_{j=1}^{n} \alpha_{j} x_{j}=0$ with $a_{j} \in \mathbb{Z}$ implies that $a_{j}=0$ for all $j$.

There are also new results $[8,38]$ for the case when $K=\cup_{j=1}^{p} K_{j}$ is a union of $p$ disjoint Jordan curves or arcs (not necessarily smooth), where quasi-smoothness or Dini-smoothness is required instead of smoothness.

Parreau-Widom sets have positive Lebesgue measure (see, e.g., [14] for a proof). All finite gap sets (see, e.g., $[15,17])$ and symmetric Cantor sets with positive length (see, e.g., [29]) are Parreau-Widom sets. Hence, in all cases considered above the sequence of Widom factors is bounded. The second and the third authors showed that any subexponential growth of $\left(W_{n}(K)\right)_{n=1}^{\infty}$ can be achieved and presented a Cantor-type set with highly irregular behaviour of Widom factors, namely [21],

1. For each $\left(M_{n}\right)$ of subexponential growth there is $K$ with $W_{n}(K) \geq M_{n}$ for all $n$.

2. Given $\sigma_{n} \searrow 0$ and $M_{n} \rightarrow \infty$ (of subexponential growth), there is $K$ such that $W_{n_{j}}(K)<2\left(1+\sigma_{n_{j}}\right)$ and $W_{m_{j}}(K)>M_{m_{j}}$ for some subsequences $\left(n_{j}\right)$ and $\left(m_{j}\right)$.

In the last section, we consider non-Parreau-Widom sets with slow growth of Widom factors.

\subsection{General Orthogonal Polynomials}

Given $\mu$ as above, the Gram-Schmidt process in $L^{2}(\mu)$ defines orthonormal polynomials $p_{n}(z, \mu)=\kappa_{n} z^{n}+\cdots$ with $\kappa_{n}>0$. Let $q_{n}=\kappa_{n}^{-1} p_{n}$. Then $\left\|q_{n}\right\|_{2}=$ $\kappa_{n}^{-1}=\inf _{Q \in \mathscr{M}_{n}}\|Q\|_{2}$. If $K \subset \mathbb{R}$, then a three-term recurrence relation

$$
x q_{n}(x)=q_{n+1}(x)+b_{n} q_{n}(x)+a_{n-1}^{2} q_{n-1}(x)
$$

is valid with the Jacobi parameters $a_{n}=\kappa_{n} / \kappa_{n+1}$ and $b_{n}=\int x p_{n}^{2}(x) \mathrm{d} \mu(x)$. Since $\mu(\mathbb{R})=1$, we have $p_{0}=q_{0} \equiv 1$, so $\kappa_{0}=1$ and $a_{0} a_{1} \cdots a_{n-1}=\kappa_{n}^{-1}$.

Thus, $W_{n}^{2}(\mu)=\left(\kappa_{n} \cdot \operatorname{Cap}^{n}(K)\right)^{-1}$ and, in particular, for $K=[-1,1]$ we have $W_{n}^{2}(\mu)=a_{0} a_{1} \cdots a_{n-1} \cdot 2^{n}$.

For example, the equilibrium measure $\mathrm{d} \mu_{[-1,1]}=\frac{\mathrm{d} x}{\pi \sqrt{1-x^{2}}}$ generates the Chebyshev polynomials of the first kind with $W_{n}^{2}\left(\mu_{[-1,1]}\right)=\sqrt{2}$ for all $n$, whereas for the Chebyshev polynomials of the second kind $\mathrm{d} \nu=\frac{2}{\pi} \sqrt{1-x^{2}} \mathrm{~d} x$ and $W_{n}^{2}(\nu)=1$.

The Jacobi parameters generate the matrix

$$
J=\left(\begin{array}{ccccc}
b_{0} & a_{0} & 0 & 0 & \ldots \\
a_{0} & b_{1} & a_{1} & 0 & \ldots \\
0 & a_{1} & b_{2} & a_{2} & \ldots \\
\vdots & \vdots & \vdots & \vdots & \ddots
\end{array}\right),
$$


where $\mu$ is the spectral measure for the unit vector $\delta_{1}$ and the self-adjoint operator $J$ on $l_{2}\left(\mathbb{Z}_{+}\right)$, which is defined by this matrix.

Both $\left(a_{n}\right)$ and $\left(b_{n}\right)$ are bounded sequences. Conversely, if we are given bounded sequences $\left(a_{n}\right)$ and $\left(b_{n}\right)$ with $a_{n}>0$ and $b_{n} \in \mathbb{R}$, then, as a result of the spectral theorem, there is a unique probability measure $\mu$ such that the associated recurrence coefficients are $\left(a_{n}, b_{n}\right)_{n=0}^{\infty}$.

For a wide class of measures the polynomials $p_{n}=p_{n}(\cdot, \mu)$ enjoy regular limit behaviour. Let $\Omega=\overline{\mathbb{C}} \backslash K$ and $v_{p_{n}}$ be the counting measure on the zeros of $p_{n}$. Suppose the set $K$ is not polar. Let us consider the asymptotics:

1. $\kappa_{n}^{1 / n} \rightarrow \operatorname{Cap}(K)^{-1}$

2. $\left|p_{n}\right|^{1 / n} \rightrightarrows \exp g_{\Omega} \quad$ (locally uniformly on $\overline{\mathbb{C}} \backslash \operatorname{Conv} \cdot h u l l(K)$ )

3. $\lim \sup \left|p_{n}(z)\right|^{1 / n} \stackrel{\text { q.e. }}{=} 1$ on $\partial \Omega$

4. $\frac{1}{n} v_{p_{n}} \stackrel{w^{*}}{\rightarrow} \mu_{K}$.

By Theorem 3.1.1 in [34], the conditions (1)-(3) are pairwise equivalent. If, in addition, $K \subset \partial \Omega$ and the minimal carrier capacity of $\mu$ is positive, then (1) is equivalent to (4).

A measure $\mu$ with support $K$ is called regular in the Stahl-Totik sense ( $\mu \in \mathbf{R e g}$ ) if (1) is valid. This definition allows measures with polar support. In this case the equivalence of (1)-(3) is still valid if we take $g_{\Omega} \equiv \infty$ in (2).

Till now there is no complete description of regularity in terms of the size of $\mu$. We will use the generalized version of the Erdös-Turán criterion for $K \subset \mathbb{R}$ ([34], Theorem 4.1.1): $\mu \in \operatorname{Reg}$ provided $\mathrm{d} \mu / \mathrm{d} \mu_{K}>0, \quad \mu_{K}-$ a.e. Thus (see also [41] and [32]), equilibrium measures are regular in the Stahl-Totik sense.

We see that $\mu \in \mathbf{R e g}$ if and only if $\left(W_{n}^{2}(\mu)\right)_{n=1}^{\infty}$ has subexponential growth.

\subsection{Strong Asymptotics}

The conditions (1)-(4) from the previous section can be considered as weak asymptotics. For measures from the Szegó class stronger asymptotics are valid for the corresponding orthogonal polynomials.

Suppose $\mathrm{d} \mu=\omega(x) \mathrm{d} x$ on $K=[-1,1]$. Then we say that $\mu$ is in the Szegó class $(\mu \in S z[-1,1])$ if

$$
I(\omega):=\int_{-1}^{1} \frac{\log \omega(x)}{\pi \sqrt{1-x^{2}}} \mathrm{~d} x=\int \log \omega(x) \mathrm{d} \mu_{K}(x)>-\infty,
$$

which means that the integral converges for it cannot be $+\infty$. For such measures [35, p. 297]

$$
p_{n}(z, \mu)=\kappa_{n} z^{n}+\cdots=(1+o(1))\left(z+\sqrt{z^{2}-1}\right)^{n} \frac{1}{\sqrt{2 \pi}} D_{\mu}^{-1}(z),
$$


where the Szegó function

$$
D_{\mu}(z)=\exp \left(\frac{1}{2} \sqrt{z^{2}-1} \int \frac{\log \left[\omega(x) \sqrt{1-x^{2}}\right]}{z-x} \mathrm{~d} \mu_{K}(x)\right)
$$

is a certain outer function in the Hardy space on $\mathbb{C} \backslash[-1,1]$. Here the square root $\sqrt{z^{2}-1}$ is taken such that $\left|z+\sqrt{z^{2}-1}\right|>1$ at $z \notin K$.

Now $z \rightarrow \infty$ implies not only that $\kappa_{n}^{1 / n} \rightarrow 2$, so $\mu \in \mathbf{R e g}$, but also the existence of

$$
\lim _{n} W_{n}^{2}(\mu)=\sqrt{\pi} \exp (I(\omega) / 2)
$$

((12.7.2) in [35]), which is essentially stronger than the fact of subexponential growth of the sequence.

The inverse implication is also valid: if $\lim _{n} W_{n}^{2}(\mu)$ exists in $(0, \infty)$, then we have $\mu \in S z[-1,1]$ (see, e.g., T.2.4 in [16]).

The Szegô theory was extended first to the case of measures that generate a finite gap Jacobi matrix (see, e.g., $[9,16,28,42]$ ) and then for measures on $\mathbb{R}$ such that the essential support of $\mu$ is a Parreau-Widom set.

Let $\left\{y_{j}\right\}_{j}$ be the set of all isolated points of the support of $\mu$ and $K=\operatorname{ess} \operatorname{supp}(\mu)$, so $\operatorname{supp}(\mu)=K \cup\left\{y_{j}\right\}_{j}$. Suppose that $K$ is a Parreau-Widom set, so it has positive Lebesgue measure. Let $\omega(x) \mathrm{d} x$ be the absolutely continuous part of $\mathrm{d} \mu$ in its Lebesgue decomposition. In addition, let $\sum g_{\mathbb{C} \backslash K}\left(y_{j}\right)<\infty$. Then, in our terms (see, e.g., Theorem 2 in [14]),

$$
\int \log \omega(x) \mathrm{d} \mu_{K}(x)>-\infty \Longleftrightarrow \limsup _{n \rightarrow \infty} W_{n}^{2}(\mu)>0 .
$$

Moreover, if one of the conditions above holds, then there is a positive number $M$ such that

$$
\frac{1}{M}<W_{n}^{2}(\mu)<M
$$

holds for all $n$. Thus, any of the conditions in (7.1) implies regularity of the corresponding measure.

We write $\mu \in S z(K)$ if the Szegô condition on the left-hand side of (7.1) is valid. We see that this definition can be applied only to measures that have nontrivial absolutely continuous part. On the other hand, the Widom condition (on the right side) is applicable to any measure.

For each Parreau-Widom set $K$, its equilibrium measure $\mu_{K}$ belongs to $S z(K)$ [14] and the sequence $\left(W_{n}^{2}\left(\mu_{K}\right)\right)$ is bounded above [18]. In [5, 7] the first two authors presented non-polar sets with unbounded above sequence $\left(W_{n}^{2}\left(\mu_{K}\right)\right)$. 
The Widom condition is the main candidate to characterize the Szegó class in the general case. In [5] it was conjectured that the equilibrium measure always is in the Szegő class and the following form of the Szegô condition was suggested

$$
\int \log \left(\mathrm{d} \mu / \mathrm{d} \mu_{K}\right) \mathrm{d} \mu_{K}(t)>-\infty
$$

that can be used for all non-polar sets.

\subsection{Widom Factors for the Hilbert Norm}

Here we consider some model examples of Widom-Hilbert factors (see [7] for more details).

1. Jacobi weight. For $-1<\alpha, \beta<\infty$ let

$$
\mathrm{d} \mu_{\alpha, \beta}=C_{\alpha, \beta}^{-1}(1-x)^{\alpha}(1+x)^{\beta} \mathrm{d} x
$$

with

$$
C_{\alpha, \beta}=\int_{-1}^{1}(1-x)^{\alpha}(1+x)^{\beta} \mathrm{d} x
$$

Set $W_{\alpha, \beta}:=\sqrt{\frac{\pi}{2^{\alpha+\beta} C_{\alpha, \beta}}}$. Then $W_{n}^{2}\left(\mu_{\alpha, \beta}\right) \rightarrow W_{\alpha, \beta}$. Here, $W_{\alpha, \beta} \rightarrow 0$ as $(\alpha, \beta)$ approaches the boundary of the domain $(-1, \infty)^{2}$ and

$$
\sup _{-1<\alpha, \beta<\infty} W_{\alpha, \beta}=W_{-1 / 2,-1 / 2}=\sqrt{2} .
$$

We see that, in the class of Jacobi polynomials, the maximal value of $I(\omega)$ is attained on the equilibrium measure. By Jensen's inequality, $\mu_{[-1,1]}$ gives the maximum of the Szegó integral in the whole class $S z[-1,1]$. Indeed,

$$
\int \log \left(\omega / \omega_{e}\right) \mathrm{d} \mu_{[-1,1]} \leq \log \int \omega / \omega_{e} \mathrm{~d} \mu_{[-1,1]}=\log \int_{-1}^{1} \omega(x) \mathrm{d} x=0,
$$

where $\mu \in S z[-1,1]$ with $\mathrm{d} \mu=\omega(x) \mathrm{d} x$ and $\omega_{e}(x)=\frac{1}{\pi \sqrt{1-x^{2}}}$.

2. Regular measure beyond the Szegô class. A typical example of such measure is given by the density

$$
\omega(x)=\frac{1+a}{2 \pi} \exp (-2 t \cdot \arcsin x) \cdot|\Gamma(1 / 2+i t)|^{2}
$$


with $t=\frac{a x+b}{2 \sqrt{1-x^{2}}}$, where $a, b \in \mathbb{R}, a \geq|b|, a+|b|>0$. The measure generates the Pollaczek polynomials. Here, $\mu$ is regular, as $\omega>0$ for $|x|<1$, but since $\omega \rightarrow 0$ exponentially fast near \pm 1 , the integral $I(\omega)$ diverges, so $\mu \notin S z[-1,1]$. In this case,

$$
\lim _{n} W_{n}^{2}(\mu) \cdot n^{a / 2}=\Gamma\left(\frac{a+1}{2}\right),
$$

so the Widom factors go to zero but not very fast.

3. $\mu \notin$ Reg. Using techniques from [34], one can show that any rate of decrease, as fast as we wish, can be achieved for the sequence $\left(W_{n}^{2}(\mu)\right)$. Namely, ([7], Example 5) for each sequence $\sigma_{n} \searrow 0$ there exists a measure $\mu$ such that $W_{n}^{2}(\mu)<\sigma_{n}$ for all $n$. Here, $\operatorname{Cap}(\operatorname{supp}(\mu))$ does not coincide with the minimal carrier capacity of $\mu$.

4. Jacobi matrix with periodic coefficients $\left(a_{n}\right)$ and zero (or slowly oscillating) main diagonal. The periodic coefficients give a Jacobi matrix in the Szegó class. We follow [26] here.

Let $a_{2 n-1}=a, a_{2 n}=b$ for $n \in \mathbb{N}$ with $b>0$ and $a=b+2$. These values with $b_{n}=0$ define a Jacobi matrix $B_{0}$ with spectrum

$$
\sigma\left(B_{0}\right)=[-b-a, b-a] \cup[a-b, a+b] .
$$

The same values $\left(a_{n}\right)_{n=1}^{\infty}$ with $b_{n}=\sin n^{\gamma}$ for $0<\gamma<1$ give a matrix $B$ with

$$
\sigma(B)=[-b-a-1, b-a+1] \cup[a-b-1, a+b+1] .
$$

Then $\operatorname{Cap}\left(\sigma\left(B_{0}\right)\right)=\sqrt{a b}, \operatorname{Cap}(\sigma(B))=\sqrt{a(b+1)}$. Let $\mu_{0}$ and $\mu$ be spectral measures for $B_{0}$ and $B$ correspondingly. Then $W_{2 n}^{2}\left(\mu_{0}\right)=1$ and $W_{2 n-1}^{2}\left(\mu_{0}\right)=$ $\sqrt{a / b}$. Hence, $\mu_{0} \in S z\left(\sigma\left(B_{0}\right)\right)$, as we expected. On the other hand,

$$
W_{2 n}^{2}(\mu)=\left(\frac{b}{b+1}\right)^{n}
$$

and

$$
W_{2 n+1}^{2}(\mu)=\left(\frac{b}{b+1}\right)^{n} \sqrt{\frac{a}{b+1}} .
$$

Thus, $W_{n}^{2}(\mu) \rightarrow 0$ as $n \rightarrow \infty, \mu \notin S z(\sigma(B))$ and, moreover, $\mu \notin$ Reg.

5. Julia sets generated by $T(z)=z^{3}-\lambda z$ with $\lambda>3$ [11].

Iterations $T_{0}=z, T_{n}=T_{n-1}(T)$ define a Cantor-type Julia set $J=\operatorname{supp}\left(\mu_{J}\right)$. Let $W_{k}:=W_{k}^{2}\left(\mu_{J}\right)$. Then $W_{3^{n}}=1$, whereas $W_{3^{n}-1} \rightarrow \infty$. Also,

$$
W_{3^{n}+1} \rightarrow \sqrt{2 \lambda / 3}, W_{3^{n}+2} \rightarrow \sqrt{2} \lambda / 3 \text {, etc. }
$$




\subsection{Weakly Equilibrium Cantor Sets}

The theory of orthogonal polynomials is well developed for measures that are absolutely continuous with respect to the Lebesgue measure $\left(\mu=\mu_{a}\right)$, at least for the finite gap case. There are also numerous results for measures $\left(\mu=\mu_{a}+\mu_{p}\right)$ that allow nontrivial point spectrum. Here in the description of the Szegó class a condition of Blaschke-type is added. But there are only a few results for concrete singular continuous measures, mainly they are concerned with orthogonal polynomials for equilibrium measures on Julia sets. As we mentioned above, Parreau-Widom sets (in particular homogeneous sets in the sense of Carleson) may have Cantor structure, but their Lebesgue measure is positive.

There are only particular results for a prescribed measure $\mu$ supported on a Cantor set with zero Lebesgue measure. For example, if $\mu$ is the Cantor-Lebesgue measure or the equilibrium measure on the Cantor ternary set $K_{0}$, then a little is known except some conjectures depending on numerical results. For this case and other attractors of iterated function systems, we refer the reader to [22, 23, 25].

The first two authors found in [5] a new family of orthogonal polynomials with respect to the equilibrium measure on the so-called weakly equilibrium Cantor sets, that were suggested in [20]. Here we recall the construction. Given $\gamma=\left(\gamma_{s}\right)_{s=1}^{\infty}$ with $0<\gamma_{s}<\frac{1}{4}$, let $r_{0}=1$ and $r_{s}=\gamma_{s} r_{s-1}^{2}$. We define recursively polynomials

$$
P_{2}(x)=x(x-1)
$$

and

$$
P_{2^{s+1}}=P_{2^{s}} \cdot\left(P_{2^{s}}+r_{s}\right)
$$

We consider the complex level domains

$$
D_{s}=\left\{z \in \mathbb{C}:\left|P_{2^{s}}(z)+r_{s} / 2\right|<r_{s} / 2\right\}
$$

with $D_{s} \searrow$, which allows, by the Harnack Principle, to get a good representation of the Green function for the intersection of domains, and

$$
E_{s}:=\left\{x \in \mathbb{R}:\left|P_{2^{s}}(x)+r_{s} / 2\right| \leq r_{s} / 2\right\}=\cup_{j=1}^{2^{s}} I_{j, s} .
$$

Then the set

$$
K(\gamma):=\bigcap_{s=1}^{\infty} \bar{D}_{s}=\bigcap_{s=1}^{\infty} E_{s}=\bigcap_{s=1}^{\infty}\left(\frac{2}{r_{s}} P_{2^{s}}+1\right)^{-1}([-1,1])
$$

is an intersection of polynomial preimages that provides some additional useful features. In particular, $P_{2^{s}}+r_{s} / 2$ is the $2^{s}$ th Chebyshev polynomial on $K(\gamma)$. 
At least for small $\gamma$, the set $K(\gamma)$ is weakly equilibrium in the following sense. Let us distribute uniformly the mass $2^{-s}$ on each $I_{j, s}$ for $1 \leq j \leq 2^{s}$. This defines a measure $\lambda_{s}$ supported on $E_{s}$ with $d \lambda_{s}=\left(2^{s} l_{j, s}\right)^{-1} \mathrm{~d} t$ on $I_{j, s}$. Then $\lambda_{s} \stackrel{*}{\rightarrow} \mu_{K(\gamma)}$ provided $\gamma_{n} \leq 1 / 32$ and $K(\gamma)$ is not polar.

In [21] the Widom-Chebyshev factors for $K(\gamma)$ were calculated and the result mentioned in Sect. 7.2 was obtained.

In [4] it was shown that, provided some restriction on the sequence $\gamma$, the equilibrium measure on $K(\gamma)$ and the corresponding Hausdorff measure are mutually absolutely continuous. This is not valid for geometrically symmetric Cantor-type sets, where these measures are essentially different. Makarov and Volberg proved in [24] a surprising result: the equilibrium measure for the classical Cantor set is supported by a set whose Hausdorff dimension is strictly smaller than $\log 2 / \log 3$. Therefore, $\mu_{K_{0}}$ is mutually singular with the Hausdorff measure of the set. Later this was generalized to Cantor-type sets of higher dimension and to Cantor repellers that appear in complex dynamics.

The set $K(\gamma)$ has positive Lebesgue measure if $\gamma_{s}$ are rather closed to $\frac{1}{4}$. Moreover, in the limit case $\gamma_{s}=\frac{1}{4}$ for all $s$ we have $K(\gamma)=[0,1]$.

\subsection{Orthogonal Polynomials on $K(\gamma)$}

The set $K(\gamma)$ is non-polar if and only if

$$
\sum_{n=1}^{\infty} 2^{-n} \log \frac{1}{\gamma_{n}}<\infty,
$$

where the series represents the Robin constant of the set. Orthogonal polynomials with respect to the equilibrium measure on non-polar $K(\gamma)$ were considered in [5]. It is proven that the monic orthogonal polynomials $Q_{2^{s}}$ coincide with the Chebyshev polynomials of the set. Procedures were suggested to find orthogonal polynomials $Q_{n}$ of all degrees and to calculate the corresponding Jacobi parameters. In addition, it was shown that the sequence of Widom factors is bounded below by a positive number (in confirmation of our hypothesis that equilibrium measures always belong to the Szegó class in its Widom characterization).

First the authors used a technique of decomposition of zeros of $P_{2^{s}}+r_{s} / 2$ into certain groups and the approximation of the equilibrium measure $\mu_{K(\gamma)}$ by the normalized counting measure at zeros of the Chebyshev polynomials of the set. Namely, let $v_{s}=2^{-s} \sum_{k=1}^{2^{s}} \delta_{x_{k}}$, where $\left(x_{k}\right)_{k=1}^{2^{s}}$ are the zeros of $P_{2^{s}}+r_{s} / 2$ (they are simple and real). Then for $s>m$ it is possible to decompose all zeros $\left(x_{k}\right)_{k=1}^{2^{s}}$ into $2^{s-m-1}$ groups, on which we can control the value of $P_{2^{m}}+r_{m} / 2$. This allows to show that

$$
\int\left(P_{2^{m}}+\frac{r_{m}}{2}\right) \mathrm{d} v_{s}=0
$$


Since $v_{s} \rightarrow \mu_{K(\gamma)}$ in the weak-star topology, we have that the integral

$$
\int\left(P_{2^{m}}+\frac{r_{m}}{2}\right) \mathrm{d} \mu_{K(\gamma)}
$$

also is zero.

Similarly it was shown that

$$
\int\left(P_{2^{i_{1}}}+\frac{r_{i_{1}}}{2}\right)\left(P_{2^{i_{2}}}+\frac{r_{i_{2}}}{2}\right) \ldots\left(P_{2^{i_{n}}}+\frac{r_{i_{n}}}{2}\right) \mathrm{d} \nu_{s}=0
$$

for $0 \leq i_{1}<i_{2}<\cdots<i_{n}<s$. Each polynomial $P$ of degree less than $2^{s}$ is a linear combination of polynomials of the type

$$
\left(P_{2^{s-1}}+\frac{r_{s-1}}{2}\right)^{n_{s-1}} \ldots\left(P_{2}+\frac{r_{1}}{2}\right)^{n_{1}}\left(x-\frac{1}{2}\right)^{n_{0}},
$$

with $n_{i} \in\{0,1\}$. Therefore, $Q_{2^{s}}$ coincides with $P_{2^{s}}+r_{s} / 2$. In addition, the norm $\left\|Q_{2^{s}}\right\|_{2}$ has a simple representation in terms of $\left(\gamma_{k}\right)_{k=1}^{s+1}((3.1)$ in [5]).

In the next step, $A$-type and $B$-type polynomials were introduced. In particular, for $2^{m} \leq n<2^{m+1}$ with the binary representation $n=i_{m} 2^{m}+\cdots+i_{0}$, the second polynomial is

$$
B_{n}=\left(Q_{2^{m}}\right)^{i_{m}}\left(Q_{2^{m-1}}\right)^{i_{m-1}} \ldots\left(Q_{1}\right)^{i_{1}}
$$

The polynomials $B_{(2 k+1) \cdot 2^{s}}$ and $B_{(2 j+1) \cdot 2^{m}}$ are orthogonal for all $j, k, m, s \in \mathbb{Z}_{+}$with $s \neq m$. They can be considered as a basis in the set of polynomials: for each $n \in \mathbb{N}$ with $n=2^{s}(2 k+1)$, the polynomial $Q_{n}$ has a unique representation as a linear combination of

$$
B_{2^{s}}, B_{3 \cdot 2^{s}}, B_{5 \cdot 2^{s}} \ldots, B_{(2 k-1) \cdot 2^{s}}, B_{(2 k+1) \cdot 2^{s}} .
$$

This allows to present formulas to express coefficients of each $Q_{n}$ and the corresponding Jacobi parameters in terms of $\left(\gamma_{k}\right)_{k=1}^{\infty}$. Some asymptotics of Jacobi parameters were presented in Theorem 4.7 in [5]: Let $\gamma_{s} \leq 1 / 6$ for all s. Then $\lim _{s \rightarrow \infty} a_{j \cdot 2^{s}+n}=a_{n}$ for $j \in N$ and $n \in Z_{+}$. Here, $a_{0}:=0$. In particular, $\lim \inf a_{n}=0$.

In the last section the Widom factors for $\mu_{K(\gamma)}$ were evaluated. If $\gamma_{k} \leq \frac{1}{6}$ for all $k$, then

$$
\liminf _{n \rightarrow \infty} W_{n}=\liminf _{s \rightarrow \infty} W_{2^{s}} \geq \sqrt{2}
$$

and

$$
\limsup _{n \rightarrow \infty} W_{n}=\infty \text {. }
$$


The following examples illustrate the behaviour of Widom factors:

1. If $\gamma_{n} \rightarrow 0$, then $W_{2^{s}} \rightarrow \infty$. Therefore $W_{n} \rightarrow \infty$.

2. There exists $\gamma_{n} \nrightarrow 0$ with $W_{n} \rightarrow \infty$. One can take $\gamma_{2 k}=1 / 6, \gamma_{2 k-1}=1 / k$.

3. If $\gamma_{n} \geq c>0$ for all $n$, then $\liminf _{n \rightarrow \infty} W_{n} \leq 1 / 2 c$.

4. There exists $\gamma$ with inf $\gamma_{n}=0$ and $\liminf _{n \rightarrow \infty} W_{n}<\infty$. Here we can take $\gamma_{n}=1 / 6$ for $n \neq n_{k}$ and $\gamma_{n_{k}}=1 / k$ for a sparse sequence $\left(n_{k}\right)_{k=1}^{\infty}$. Then $\left(W_{2^{n_{k}}}\right)_{k=1}^{\infty}$ is bounded.

Later, in [6], it was shown that $K(\gamma)$ is a Parreau-Widom set if and only if

$$
\sum_{n=1}^{\infty} \sqrt{\frac{1}{4}-\gamma_{n}}<\infty .
$$

\subsection{Generalized Julia Sets}

In [6] the first two authors generalized some of the results [10-12] by Barnsley et al. obtained for autonomous Julia sets to more general class of sets. Also, [6] is a generalization of Alpan and Goncharov [5] as $K(\gamma)$ can be considered as a generalized Julia set.

We recall some basic definitions.

Let $\left(f_{n}(z)\right)_{n=1}^{\infty}$ be a sequence of rational functions with $\operatorname{deg} f_{n} \geq 2$. in $\overline{\mathbb{C}}$. Let us define $F_{n}(z):=f_{n} \circ F_{n-1}(z)$ recursively for $n \geq 1$ and $F_{0}(z)=z$. Then domain of normality for $\left(F_{n}\right)_{n=1}^{\infty}$ in the sense of Montel is called the Fatou set for $\left(f_{n}\right)$. The complement of the Fatou set in $\overline{\mathbb{C}}$ is called the Julia set for $\left(f_{n}\right)$. We denote them by $F_{\left(f_{n}\right)}$ and $J_{\left(f_{n}\right)}$, respectively. In particular, if $f_{n}=f$ for some fixed rational $f$ for all $n$, then we use the notations $F(f)$ and $J(f)$. To distinguish this last case, the word autonomous is used.

We consider only polynomial Julia sets. In order to have an appropriate Julia set in terms of orthogonal polynomials and potential theory, we need to put some restrictions on the given polynomials. Let $f_{n}(z)=\sum_{j=0}^{d_{n}} a_{n, j} \cdot z^{j}$ where $d_{n} \geq 2$ and $a_{n, d_{n}} \neq 0$ for all $n \in \mathbb{N}$. Following [13], we say that $\left(f_{n}\right)$ is a regular polynomial sequence if the following properties are satisfied:

- There exists a real number $A_{1}>0$ such that $\left|a_{n, d_{n}}\right| \geq A_{1}$, for all $n \in \mathbb{N}$.

- There exists a real number $A_{2} \geq 0$ such that $\left|a_{n, j}\right| \leq A_{2}\left|a_{n, d_{n}}\right|$ for $j=$ $0,1, \ldots, d_{n}-1$ and $n \in \mathbb{N}$.

- There exists a real number $A_{3}$ such that

$$
\log \left|a_{n, d_{n}}\right| \leq A_{3} \cdot d_{n},
$$

for all $n \in \mathbb{N}$. 
If $\left(f_{n}\right)$ is a regular polynomial sequence, then we write $\left(f_{n}\right) \in \mathscr{R}$. If this is the case then, by Brück and Büger [13], $J_{\left(f_{n}\right)}$ is a compact subset of $\mathbb{C}$ that is regular with respect to the Dirichlet problem. Thus, $\operatorname{Cap}\left(J_{\left(f_{n}\right)}\right)>0$. Moreover, $J_{\left(f_{n}\right)}$ is just the boundary of

$$
\mathscr{A}_{\left(f_{n}\right)}(\infty):=\left\{z \in \overline{\mathbb{C}}:\left(F_{n}(z)\right)_{n=1}^{\infty} \text { goes locally uniformly to } \infty\right\} .
$$

Let $K=J_{\left(f_{n}\right)}$ with $\left(f_{n}\right) \in \mathscr{R}$. In [6], it was shown that, for each integer $n$, the monic orthogonal polynomial associated with $\mu_{K}$ of degree $d_{1} \cdots d_{n}$ can be written explicitly in terms of $F_{n}$. In [3], it was proven that the Chebyshev polynomials of degree $d_{1} \cdots d_{n}$ on $K$ are same up to constant terms with the orthogonal polynomials for $\mu_{K}$.

In some cases the set $J_{\left(f_{n}\right)}$ is real. For example, this is valid for admissible (in the sense of Geronimo and Van Assche [19]) polynomials. Then a three-term recurrence relation is valid for orthogonal polynomials and the corresponding Jacobi coefficients can be found by a recursive procedure that is depicted.

Let a sequence $\gamma$ be the same as in Sect. 7.6. If we take

$$
f_{n}(z)=\frac{1}{2 \gamma_{n}}\left(z^{2}-1\right)+1
$$

for all $n$, then $K_{1}(\gamma):=J_{\left(f_{n}\right)}$ is a stretched version of the set $K(\gamma)$. Let $\varepsilon_{k}=\frac{1}{4}-\gamma_{k}$.

By Theorem 8 in [6], the Green function $g_{\mathbb{C} \backslash K_{1}(\gamma)}$ has optimal smoothness (is Hölder continuous with the exponent $1 / 2$ ) if and only if $\sum_{k=1}^{\infty} \varepsilon_{k}<\infty$. This completes the analysis of smoothness of $g_{\mathbb{C} \backslash K(\gamma)}$ for the case of small $\gamma$ in [20].

By Theorem 9 in [6], $K_{1}(\gamma)$ is a Parreau-Widom set if and only if $\sum_{k=1}^{\infty} \sqrt{\varepsilon_{k}}<\infty$.

It is interesting to analyse the character of growth of Widom's factor for nonParreau-Widom sets.

\subsection{Widom's Factor for Non-Parreau-Widom Sets}

Here we return to Widom factors for the Chebyshev norm on $K(\gamma)$. As above, let $\varepsilon_{k}=\frac{1}{4}-\gamma_{k}$. Clearly, $0<1-4 \varepsilon_{k}<1$. Suppose

$$
\sum_{k=1}^{\infty} \varepsilon_{k}<\infty \text { but } \sum_{k=1}^{\infty} \sqrt{\varepsilon_{k}}=\infty
$$

By $C$ we denote the product $2 \prod_{k=1}^{\infty}\left(1-4 \varepsilon_{k}\right)^{-1}$, which is finite by (7.2). Also this condition implies that the set $K(\gamma)$ is not polar and is not Parreau-Widom. 
Theorem 7.1. Let $\gamma=\left(\gamma_{k}\right)_{k=1}^{\infty}$ be a monotone sequence satisfying (7.2). Then the bound $W_{n}(K(\gamma)) \leq$ Cn holds for all $n \in \mathbb{N}$.

Proof. By [21], for all $s \in \mathbb{Z}_{+}$we have

$$
W_{2^{s}}(K(\gamma))=\frac{1}{2} \exp \left(2^{s} \sum_{k=s+1}^{\infty} 2^{-k} \log \frac{1}{\gamma_{k}}\right) .
$$

Since $\left(\gamma_{k}\right)_{k=1}^{\infty}$ monotonically increases, we get the inequality

$$
W_{2^{s}}(K(\gamma)) \leq \frac{1}{2 \gamma_{s+1}}=\frac{2}{1-4 \varepsilon_{s+1}} .
$$

Given $n \in \mathbb{N}$, take $s \in \mathbb{Z}_{+}$with $2^{s} \leq n<2^{s+1}$. If $n=2^{s}$ then, by (7.3),

$$
W_{n}(K(\gamma)) \leq \frac{2}{1-4 \varepsilon_{s+1}}<C .
$$

If $n \neq 2^{s}$, then there are integer numbers $0 \leq p_{1}<p_{2}<\cdots<p_{m} \leq s-1$ with $m \leq s$ such that $n=2^{s}+2^{p_{m}}+\cdots+2^{p_{1}}$. Widom factors are logarithmic subadditive, that is $W_{n+r}(K) \leq W_{n}(K) \cdot W_{r}(K)$. Therefore,

$$
W_{n}(K(\gamma)) \leq W_{2^{s}}(K(\gamma)) \cdot W_{2^{p_{m}}}(K(\gamma)) \cdots W_{2^{p_{1}}}(K(\gamma)) .
$$

By (7.3) we see that

$$
\begin{aligned}
W_{n}(K(\gamma)) & \leq \frac{2}{1-4 \varepsilon_{s+1}} \frac{2}{1-4 \varepsilon_{p_{m}+1}} \cdots \frac{2}{1-4 \varepsilon_{p_{1}+1}} \\
& \leq 2^{s+1} C / 2<n C .
\end{aligned}
$$

This completes the proof.

Acknowledgements The first two authors are partially supported by TÜBİTAK (Scientific and Technological Research Council of Turkey), Project 115F199.

\section{References}

1. N. Achyeser, Über einige Funktionen, welche in zwei gegebenen Interwallen am wenigsten von Null abweichen. I Teil, Bulletin de l'Académie des Sciences de l'URSS. Classe des sciences mathématiques et na (9), 1163-1202 (1932)

2. N. Achyeser, Über einige Funktionen, welche in zwei gegebenen Intervallen am wenigsten von Null abweichen. II Teil, Bulletin de l'Académie des Sciences de l'URSS. Classe des sciences mathématiques et na (3), 309-344 (1933) 
3. G. Alpan, Chebyshev polynomials on generalized Julia sets. Comput. Methods Funct. Theory (2015). doi:10.1007/s40315-015-0145-8

4. G. Alpan, A. Goncharov, Two measures on Cantor sets. J. Approx. Theory 186, $28-32$ (2014)

5. G. Alpan, A. Goncharov, Orthogonal polynomials for the weakly equilibrium Cantor sets. Proc. Am. Math. Soc. (accepted for publication)

6. G. Alpan, A. Goncharov, Orthogonal polynomials on generalized Julia sets (preprint). arXiv: $1503.07098 \mathrm{v} 3$

7. G. Alpan, A. Goncharov, Widom Factors for the Hilbert Norm, vol. 107 (Banach Center Publications), pp. 9-16

8. V.V. Andrievskii, Chebyshev Polynomials on a system of continua. Constr. Approx. (2015). doi: $10.1007 / \mathrm{s} 00365-015-9280-8$

9. A.I. Aptekarev, Asymptotic properties of polynomials orthogonal on a system of contours, and periodic motions of Toda lattices. Mat. Sb. 125, 231-258 (1984). English translations in Math. USSR Sb. 53 (1986), 233-260

10. M.F. Barnsley, J.S. Geronimo, A.N. Harrington, Orthogonal polynomials associated with invariant measures on Julia sets. Bull. Am. Math. Soc. 7, 381-384 (1982)

11. M.F. Barnsley, J.S. Geronimo, A.N. Harrington, Infinite-dimensional Jacobi matrices associated with Julia sets. Proc. Am. Math. Soc. 88, 625-630 (1983)

12. M.F. Barnsley, J.S. Geronimo, A.N. Harrington, Almost periodic Jacobi matrices associated with Julia sets for polynomials. Commun. Math. Phys. 99, 303-317 (1985)

13. R. Brück, M. Büger, Generalized iteration. Comput. Methods Funct. Theory 3, 201-252 (2003)

14. J.S. Christiansen, Szegő's theorem on Parreau-Widom sets. Adv. Math. 229, 1180-1204 (2012)

15. J.S. Christiansen, B. Simon, M. Zinchenko, Finite gap Jacobi matrices. I. The isospectral torus. Constr. Approx. 32, 1-65 (2010)

16. J.S. Christiansen, B. Simon, M. Zinchenko, Finite gap Jacobi matrices, II. The Szegö class. Constr. Approx. 33, 365-403 (2011)

17. J.S. Christiansen, B. Simon, M. Zinchenko, Finite gap Jacobi matrices: a review, in Proceedings of Symposia in Pure Mathematics (American Mathematical Society, Providence, RI, 2013), pp. 87-103

18. J.S. Christiansen, B. Simon, M. Zinchenko, Asymptotics of Chebyshev polynomials, I. Subsets of $R$ (2015). http://arxiv.org/pdf/1505.02604v1.pdf

19. J.S. Geronimo, W. Van Assche, Orthogonal polynomials on several intervals via a polynomial mapping. Trans. Am. Math. Soc. 308, 559-581 (1988)

20. A. Goncharov, Weakly equilibrium Cantor type sets. Potential Anal. 40, 143-161 (2014)

21. A. Goncharov, B. Hatinoğlu, Widom factors. Potential Anal. 42, 671-680 (2015)

22. S.M. Heilman, P. Owrutsky, R. Strichartz, Orthogonal polynomials with respect to self-similar measures. Exp. Math. 20, 238-259 (2011)

23. H. Krüger, B. Simon, Cantor polynomials and some related classes of OPRL. J. Approx. Theory 191, 71-93 (2015)

24. N.G. Makarov, A.L. Volberg, On the harmonic measure of discontinuous fractals. LOMI Preprint, E-6-86, Steklov Mathematical Institute, Leningrad (1986)

25. G. Mantica, A stable Stieltjes technique for computing orthogonal polynomials and Jacobi matrices associated with a class of singular measures. Constr. Approx. 12, 509-530 (1996)

26. C. Martínez, The spectrum of periodic Jacobi matrices with slowly oscillating diagonal terms. Proc. Edinb. Math. Soc. 51, 751-763 (2008)

27. J. Milnor, Dynamics in One Complex Variables. Annals of Mathematics Studies, vol. 160 (Princeton University Press, Princeton, NJ, 2006)

28. F. Peherstorfer, Orthogonal and extremal polynomials on several intervals. J. Comput. Appl. Math. 48, 187-205 (1993)

29. F. Peherstorfer, P. Yuditskii, Asymptotic behavior of polynomials orthonormal on a homogeneous set. J. Anal. Math. 89, 113-154 (2003)

30. T. Ransford, Potential Theory in the Complex Plane (Cambridge University Press, Cambridge, 1995) 
31. K. Schiefermayr, A lower bound for the minimum deviation of the Chebyshev polynomial on a compact real set. East J. Approx. 14, 223-233 (2008)

32. B. Simon, Equilibrium measures and capacities in spectral theory. Inverse Probl. Imaging 1, 713-772 (2007)

33. B. Simon, Szegö's Theorem and Its Descendants: Spectral Theory for $L^{2}$ Perturbations of Orthogonal Polynomials (Princeton University Press, Princeton, NJ, 2011)

34. H. Stahl, V. Totik, General Orthogonal Polynomials. Encyclopedia of Mathematics, vol. 43 (Cambridge University Press, New York, 1992)

35. G. Szegő, Orthogonal Polynomials, 4th edn. (American Mathematical Society, Providence, RI, 1975). American Mathematical Society, Colloquium Publications, vol. XXIII

36. J.P. Thiran, C. Detaille, Chebyshev polynomials on circular arcs and in the complex plane, in Progress in Approximation Theory (Academic, Boston, MA, 1991), pp. 771-786

37. V. Totik, Orthogonal polynomials. Surv. Approx. Theory 1, 70-125 (2005)

38. V. Totik, T. Varga, Chebyshev and fast decreasing polynomials. Proc. Lond. Math. Soc. (2015). doi:10.1112/plms/pdv014

39. V. Totik, P. Yuditskii, On a conjecture of Widom. J. Approx. Theory 190, 50-61 (2015)

40. W. Van Assche, Asymptotics for Orthogonal Polynomials. Lecture Notes in Mathematics, vol. 1265 (Springer, Berlin, 1987)

41. H. Widom, Polynomials associated with measures in the complex plane. J. Math. Mech. 16, 997-1013 (1967)

42. H. Widom, Extremal polynomials associated with a system of curves in the complex plane. Adv. Math. 3, 127-232 (1969) 